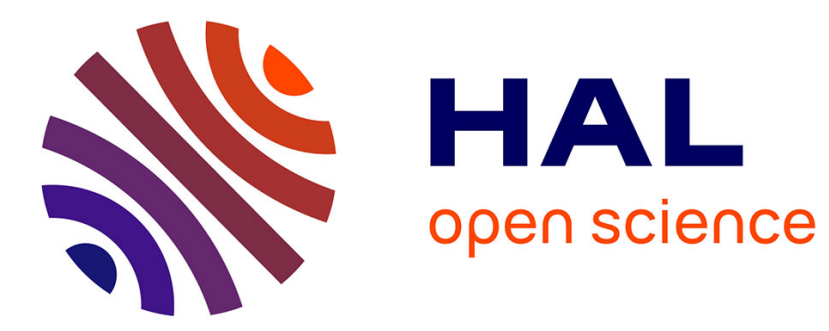

\title{
Control of the flux distribution on a solar tower receiver using an optimized aiming point strategy: Application to THEMIS solar tower
}

Adrien Salomé, Fabien Chhel, Gilles Flamant, Alain Ferrière, Frederik Thiery

\section{- To cite this version:}

Adrien Salomé, Fabien Chhel, Gilles Flamant, Alain Ferrière, Frederik Thiery. Control of the flux distribution on a solar tower receiver using an optimized aiming point strategy: Application to THEMIS solar tower. Solar Energy, 2013, 94, pp.352 - 366. 10.1016/j.solener.2013.02.025 . hal-03368303

\section{HAL Id: hal-03368303 https://hal.science/hal-03368303}

Submitted on 7 Oct 2021

HAL is a multi-disciplinary open access archive for the deposit and dissemination of scientific research documents, whether they are published or not. The documents may come from teaching and research institutions in France or abroad, or from public or private research centers.
L'archive ouverte pluridisciplinaire HAL, est destinée au dépôt et à la diffusion de documents scientifiques de niveau recherche, publiés ou non, émanant des établissements d'enseignement et de recherche français ou étrangers, des laboratoires publics ou privés. 


\title{
Control of the flux distribution on a solar tower receiver using an optimized aiming point strategy: Application to THEMIS solar tower
}

\author{
Adrien Salomé ${ }^{\mathrm{a}, \mathrm{b}, *}$, Fabien $\mathrm{Chhel}^{\mathrm{c}}$, Gilles Flamant ${ }^{\mathrm{b}}$, Alain Ferrière ${ }^{\mathrm{b}}$, Frederik Thiery ${ }^{\mathrm{a}}$ \\ a Processes, Materials and Solar Energy Laboratory, PROMES CNRS UPR8521, University of Perpignan Via Domitia (UPVD), \\ Rambla de la Thermodynamique, Tecnosud, 66100 Perpignan, France \\ ${ }^{\mathrm{b}}$ Processes, Materials and Solar Energy Laboratory, PROMES CNRS UPR8521, 7 Rue du Four Solaire, 66120 Font Romeu, France \\ ${ }^{\mathrm{c}}$ LERIA, University of Angers, 2, Boulevard Lavoisier, 49045 Angers, France
}

Received 19 June 2012; received in revised form 30 January 2013; accepted 10 February 2013

Available online 28 March 2013

Communicated by: Associate Editor L. Vant-Hull

\begin{abstract}
Life time of components is one of the technological bottle-necks in the development of solar tower power plant technology. The receiver, which is subjected to high and variable concentrated solar flux density is particularly affected: High, variable and non-homogeneous solar flux on the solar receiver walls results in strong stresses because of high temperatures, thermal shocks and temperature gradient that contribute to the reduction of the life time of this key component. This work aims to present an open loop approach to control the flux density distribution delivered on a flat plate receiver for a solar power tower. Various distributions of aiming points on the aperture of the receiver are considered. The flux density distribution on the aperture is simulated by a computer code. A specific neighborhood is defined for the TABU optimization meta-heuristic according to the size of the image of each individual heliostat. This modified algorithm is implemented to select the best aiming point for each heliostat. This approach has been validated using the example of THEMIS solar power tower in Targasonne, France.
\end{abstract}

(C) 2013 Elsevier Ltd. All rights reserved.

Keywords: Aiming point strategy; Solar power tower; Solar receiver; Open loop control

\section{Introduction}

One of the challenges for future solar tower power plants is the cost reduction of solar electricity. One option

\footnotetext{
Abbreviations: OAPS, optimized aiming point strategy; NREL, National Renewable Energy Laboratory; AP, aiming point; H, Heliostat; $m$, mirror; $M$, module; $S L$, spillage losses.

* Corresponding author at: Processes, Materials and Solar Energy Laboratory, PROMES CNRS UPR8521, University of Perpignan Via Domitia (UPVD), Rambla de la Thermodynamique, Tecnosud, 66100 Perpignan, France. Tel.: +33 46868222; fax: +33468682213.

E-mail address: adrien.salome@promes.cnrs.fr (A. Salomé).
}

consists of increasing the life time of each of the plant's components. Increasing the receiver thermal efficiency is also a relevant objective to reduce the cost of electricity. Elevated temperature gradients over the surface of the receiver and local over-heating points are clearly responsible for major degradations of this component and for significant reduction of its thermal efficiency. The distribution of temperature on the surface of the receiver mainly depends on the specific geometry of the absorber, on the manufacturing materials, on the specific heat transfer performances between the absorber and the heat transfer fluid, and on the distribution of concentrated solar 


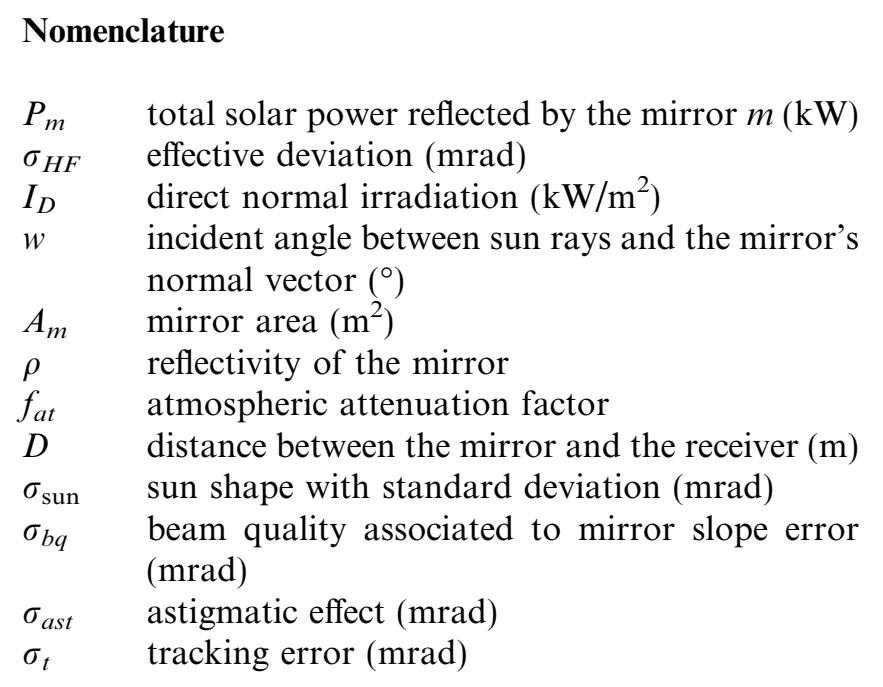

flux on the absorber. The first three of these four influential parameters are strongly dependent on the design of the receiver. The latter is much more generic, it mainly depends on the design of the solar field: height and size of the receiver aperture, layout and optics of the heliostats, and management of the tracking. Our approach aims at improving the management of the tracking, for any given optical characteristics and for a given aperture, through the selection and the implementation of an optimized aiming point strategy. The objective is to flatten as much as possible the distribution of concentrated solar flux onto the aperture of the receiver without increasing too much the spillage.

Usually the entire heliostat field aims at a single aiming point which is centered in the aperture. This latter therefore intercepts the maximum amount of power. But the concentrated solar flux distribution on the receiver very often presents a Gaussian-like distribution shape with a high peak flux density in the center.

In a previous work done at the Plataforma Solar de Almeria in Spain, a closed loop control method was developed by Garcia-Martin et al. (1999). It uses five aiming points. The receiver temperature is measured in several locations using thermocouples. The method changes the position of aiming points for each individual heliostat during operation according to the measured temperature distribution. When the temperature reaches inappropriate or out of tolerances values in a given area, the new strategy transfers power from this area to low temperature areas. This method is relevant and successful, but it only allows for operation conditions with respect to temperature thresholds since it is not based on an optimization process.

Our present work is precisely devoted to the research of an optimized flux density distribution in the aperture of the receiver according to an open loop process, independently from the measurement of temperature or any other thermal characteristics of the receiver. No experimental flux mapping is required. rec angle between the reflected beam from the center of the module and the receiver's normal $\left({ }^{\circ}\right)$

$P_{M} \quad$ total solar power reflected by the module $M$ $(\mathrm{kW})$

$|S| \quad$ size of the solution space

$n_{\mathrm{AP}}$ number of aiming point for the optimization process

$n_{\mathrm{Hel}} \quad$ number of heliostat for the optimization process

$F_{\text {max }}$ maximum flux concentration, $\left(\mathrm{kW} / \mathrm{m}^{2}\right)$

$F_{\text {min }} \quad$ minimum flux concentration $\left(\mathrm{kW} / \mathrm{m}^{2}\right)$

$P_{r e f} \quad$ power intercepted by the aperture with aiming point strategy $(\mathrm{kW})$

$P_{\text {rec }} \quad$ power intercepted by the aperture without aiming point strategy $(\mathrm{kW})$
It must be noted that when flux mapping is available on site, then a close-loop control featuring a real-time computation method might be proposed as a more robust and more reliable process.

For now the method is based on a database containing the optical characteristics of each individual heliostat. Second, the user defines a set of aiming points which forms a grid on the aperture plane. Finally the optimization process computes the best aiming point strategy which yields to a flat distribution of concentrated solar flux within a limit for spillage. The process produces a great number of solar flux distributions in the aperture plane by superposing simulated individual flux distributions delivered by each heliostat. Every individual flux distribution must be realistic. For this reason, a special attention was paid to the validation of our model through the confrontation of simulated results and experimental measurements.

The solar field and the tower at THEMIS research platform in Targasonne (France) are the supports used to illustrate and to validate our method.

The SOLTRACE software, developed by NREL (Wendelin, 2003) for the calculation of flux distribution maps has been successfully implemented and used for simulating the THEMIS solar field in a previous work done at PROMES, France (Garcia et al., 2008). This simulation code is accurate and reliable, but it requires a long computation time as it uses a ray tracing method. Therefore it is not suited for a real time control process in which a great number of flux calculations are made successively. To mitigate this disadvantage, another computing method was selected and implemented. The HFCAL model has been adapted to THEMIS heliostats' design for the calculation of flux distribution maps delivered by the entire field. HFCAL is a numerical method to calculate flux distribution maps through the calculation of two parameters: the amount of power reflected by the heliostat and a global deviation which includes Gaussian standard deviation from the sun shape, the beam reflection onto the heliostat, 
the tracking error and the astigmatic effect. The algorithm is developed on the MATLAB interface. The great advantage of this method is that a calculation takes a few seconds. A validation of this model is presented in Section 2. Section 3 addresses the aiming point optimization problem. An optimization solver is proposed. It is based on the TABU meta-heuristic method with some improvements related to the optics of the heliostats. TABU meta-heuristic, developed by Fred Glover in 1986, is a local search method used for mathematical optimization. A local search aims at finding one of the optimal solutions by taking a potential solution to a problem, and checks its immediate neighbors in the hope of finding an improved solution. TABU meta-heuristic enhances the performance of that technique by using a memory structure of the tested neighbors. The results of the optimization process are discussed and a parametric study for the optimization solver is presented in Section 4.

\section{Optical characterization of the heliostats}

It is assumed that no experimental measurement of the flux density distribution provided by the entire solar field onto the receiver is available. This distribution is therefore simulated. An optical model of THEMIS field has been developed at PROMES laboratory using SOLTRACE software. This model is used as reference to generate flux density distribution. One heliostat is composed of nine spherical mirror elements installed onto a parabolic supporting structure. The size, the curvature, the position, the orientation of these facets and the focal length of the supporting structure are known from the design specifications of each individual heliostat. In practice, deviations in the orientation, in the curvature and non-specularity of the mirrors result in a modification of the design flux distribution. A single parameter including spherical mirror elements orientation errors, curvature and non-specularity errors, and the heliostat tracking errors has been evaluated for each module of the heliostat through the experimental analysis made by image processing. A white diffuse target is placed at the top of the tower, in the receiver aperture plane. On the ground in the middle of the solar field a high resolution CCD camera with adapted filters takes a picture of the beam which is reflected onto the target (see Fig. 1). This is repeated along 1 day in order to get a series of pictures related to widely distributed locations of the sun. An image processing tool yields flux density distribution for each picture. The single optical error parameter is introduced in the simulation computer code SOLTRACE as a standard deviation in the curvature of each point of the module. This parameter is identified through the comparison of the experimental maps and the simulated ones in an iterative process (Albert, 2009). Finally, each heliostat features an individual error parameter situated in the range $0.5-2 \mathrm{mrad}$. This error parameter permitted to build an optical model of THEMIS very close to the real behaviors of heliostats.
Another experiment has been carried out to validate the utilization of a simulation code to generate the flux density distribution delivered by the solar field under various configurations of the aiming point strategy. The validation consists in comparing the simulated and the measured flux density using the image processing approach presented before. Since the measurement target cannot sustain the solar beam reflected by more than two heliostats, the experiment has been realized using the light of the moon during a full moon night. It has been assumed for simulation that moon shape was similar to sun shape. A single centered aiming point strategy and a nine aiming points strategy (square grid: 3 rows $\times 3$ columns, vertical and horizontal distance between aiming points: $0.5 \mathrm{~m}$ ) were successively applied. The complete solar field features 107 heliostats. In order to simplify the aiming point strategy control, a sample of 30 heliostats was selected, achieving a fairly good representation of the complete field. For one position of the moon two pictures are taken, each being related to one of the two aiming point strategies commented before. The corresponding flux density distributions are then compared to simulated ones in the same conditions. This was repeated at various times during the night so more than 10 different positions of the moon were considered. An example of experimental and simulated flux density distributions delivered on the measurement target for both aiming point strategies are presented in Fig. 2. We observe that the simulated flux distribution is close to the measured one for both the calculated peak flux and shape of the image. This shows that the modeling of the heliostats correctly considers the whole optical errors. The impact of an extended aiming point strategy $(3 \times 3$ aiming points $)$ on the flux density distribution is clearly observed when comparing Fig. 2a with c, and Fig. $2 \mathrm{~b}$ with $\mathrm{d}$ respectively. The size of the image is almost unchanged, but the peak flux density is decreased by $33 \%$, from $300 \mathrm{~kW} / \mathrm{m}^{2}$ for the centered aiming point ( $2 \mathrm{a}$ and $2 \mathrm{~b}$ ) to $200 \mathrm{~kW} / \mathrm{m}^{2}$ for the nine aiming points strategy ( $2 \mathrm{c}$ and $2 \mathrm{~d}$ ).

\section{Implementation of a simplified optical model using HFCAL}

\subsection{Methodology}

An aiming point strategy is destined to serve in the control system of the plant, either as a separated virtual assistant for the operator, or as a routine integrated in the automatic regulation system. In both situations, the control of the aiming point strategy in real time requires a fast calculation of the flux density distribution. SOLTRACE, like most ray tracing methods, requires a long computation time. In addition using SOLTRACE implies building an interface between SOLTRACE software and the optimization process developed on the MATLAB environment. To improve calculation time, it appears more efficient to develop an optical model on the MATLAB calculation environment too. 

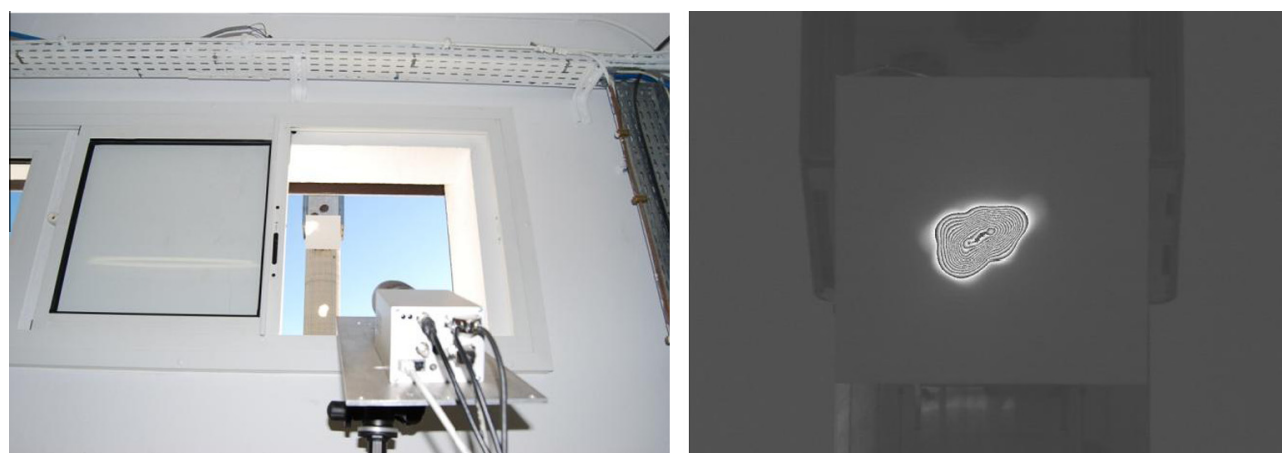

Fig. 1. Left: high resolution CCD camera used to take picture of the measurement target at THEMIS solar tower (in the background). Right: image contour of solar beam concentrated on the measurement target by heliostat B07, on August 28, 2009 at 11.46 am.

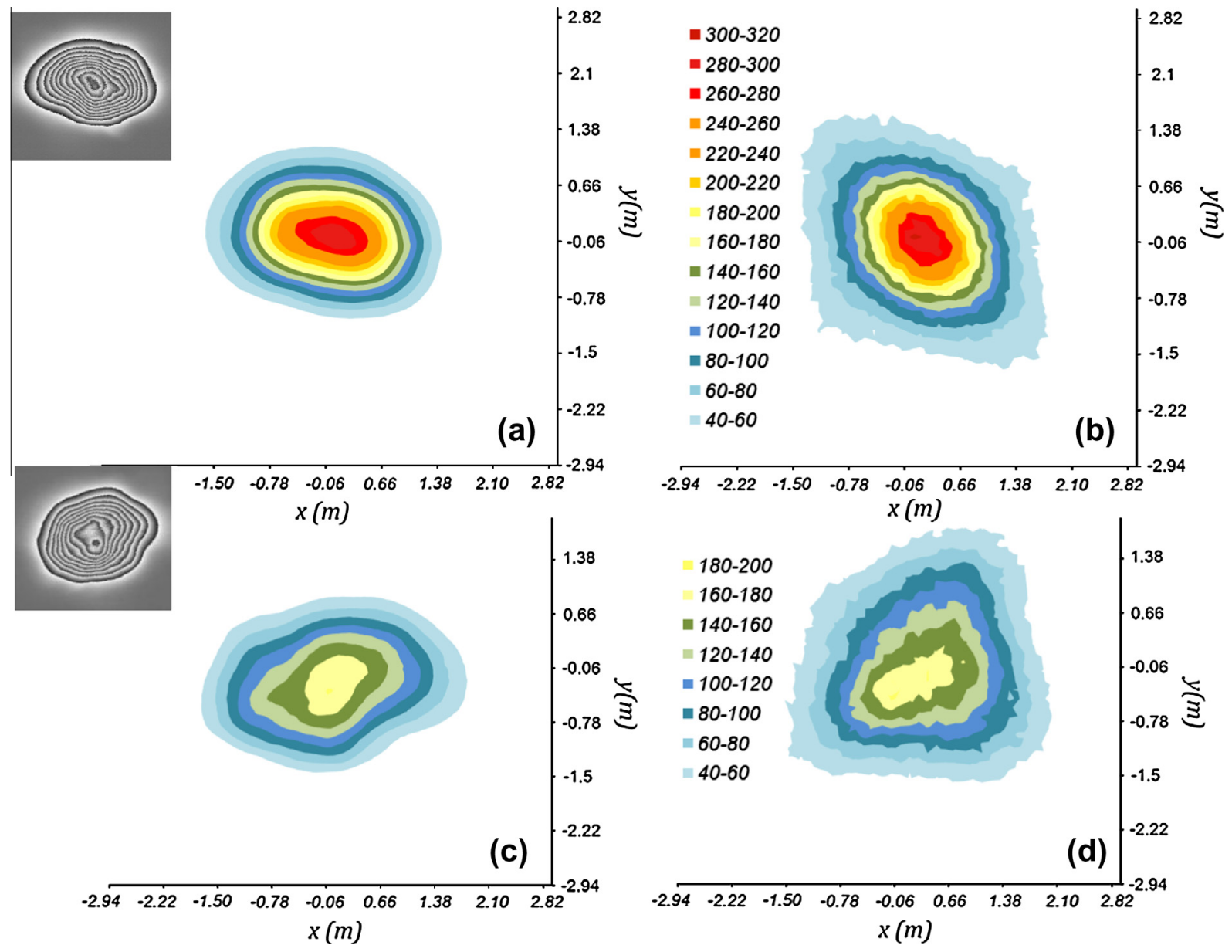

Fig. 2. Experimental (a and c) and simulated (b and d) flux density distribution on the measurement target for the full moon night of January 9 with 30 heliostats aiming at a single centered aim point ( $\mathrm{a}$ and $\mathrm{b}$ ) and using a nine aiming points strategy (c and d). Unit is $\mathrm{kW} / \mathrm{m}^{2}$, assuming $90 \%$ reflectance of the mirrors and $1000 \mathrm{~W} / \mathrm{m}^{2}$ for the direct radiation.

HFCAL model (Schwarzbözl et al., 2009) claims attractive characteristics for this application: sufficient accuracy and fast computation time (one calculation takes a few seconds against nearly a minute with SOLTRACE for the same resolution). For this reason, HFCAL simplified method was selected and was implemented. The modeling of THEMIS heliostats with SOL-
TRACE is considered as the reference model for the validation of HFCAL model. The validation is made in two steps: the flux density distribution delivered by one single heliostat is simulated by both models and the results are compared. Then the flux density distribution delivered by the entire solar field is simulated and compared. 


\subsection{Simulation of the flux distribution delivered by one heliostat using HFCAL}

The HFCAL method uses a circular Gaussian flux density function (Collado, 2010) to calculate the distribution of flux density on plane receivers. For one curvated mirror $m$ :

$\operatorname{FLUX}_{H F}(x, y)=\frac{P_{m}}{2 \pi \sigma_{H F}^{2}} e^{-\frac{\left(\left(x-x_{\mathrm{AP}}\right)^{2}+\left(y-y_{\mathrm{AP}}\right)^{2}\right)}{2 \sigma_{H F}^{2}}}$

where $P_{m}$ is the total solar power reflected by the mirror $m$ and $\sigma_{H F}$ is the effective deviation and ( $\left.x_{\mathrm{AP}}, y_{\mathrm{AP}}\right)$ the coordinates of the impact on the receiver of the ray reflected by the center of the mirror (or aiming point AP). $P_{m}$ is expressed as:

$P_{m}=I_{D} \cdot A_{m} \cdot \cos w \cdot f_{a t} \cdot \rho$

where $I_{D}$ is the DNI $\left(\mathrm{kW} / \mathrm{m}^{2}\right), A_{m}$ is the mirror area, $\cos w$ is the cosine of the incident angle between sun rays and the mirror's normal vector, $\rho$ is the reflectivity of the mirror, $f_{a t}$ is the atmospheric attenuation factor. $f_{a t}$ depends on the distance $D$ between the mirror and the receiver.

The effective deviation $\sigma_{H F}$ (Schwarzbözl et al., 2009) is the result of the convolution of the four Gaussian distribution functions considered, namely, the sun shape with standard deviation $\sigma_{\text {sun }}$, the beam quality $\sigma_{b q}$ associated to mirror slope errors (which in our case includes, as in the SOLTRACE model: curvature and non-specularity errors, spherical mirrors elements orientation errors and the influence of the heliostat tracking errors on the flux distribution), the extra deformation of the reflected ray if the incident ray is not parallel to the mirrors normal or astigmatic effect $\sigma_{\text {ast }}$, and finally the tracking error $\sigma_{t}$ that we assumed equal to $0 \mathrm{mrad}$ in both azimuth and elevation. In the HFCAL model, heliostats tracking errors $\sigma_{t}$ leads to a deviation in the distribution of flux density on plane receivers. As we explained in part 2, this deviation is included in $\sigma_{b q}$ which contains the whole optical mirrors defaults. In fact, tracking errors also lead to a translation of the whole distribution of the heliostat reflected flux on the receiver, thus, THEMIS heliostats tracking errors $(0.26 \mathrm{mrad})$ are used for the calculation of the coordinates of impact points $\left(x_{\mathrm{AP}}, y_{\mathrm{AP}}\right)$.

$\sigma_{H F}=\sqrt{D^{2}\left(\sigma_{\text {sun }}^{2}+\sigma_{b q}^{2}+\sigma_{\text {ast }}^{2}+\sigma_{t}^{2}\right)} / \sqrt{\cos r e c}$

where cos rec is the cosine of the angle between the reflected beam from the center of the module and the receiver's normal. The astigmatic effect is associated to offaxis concentration of flux. It is a function of the incident angle, of the design and the size of the heliostat, and of the ratio between focal length of the heliostat and its distance to the receiver. Although this effect corresponds to a geometrical deformation, it is treated as a standard deviation in the HFCAL model for the extra deformation of the image in both sagittal (defined by the incident and the reflected ray) and tangential plane while there is off-axis reflection (always the case for central receivers). As the HFCAL model assumes a circular normal distribution of the image, those deformations are converted to an average circular deformation described by the standard deviation $\sigma_{\text {ast }}$.

At THEMIS each heliostat $\left(53.85 \mathrm{~m}^{2}\right)$ is composed of nine modules: eight main modules of $3.62 \mathrm{~m} \times 1.794 \mathrm{~m}$, and one module of $2.46 \mathrm{~m} \times 0.828 \mathrm{~m}$ (Fig. 3). The shape of each module is approximately a portion of sphere, defined by one focal length. In practice, all the modules of one heliostat have the same focal length, which is higher than the distance to the receiver aperture. The 107 heliostats of the THEMIS solar field feature three different focal lengths for their modules: $100 \mathrm{~m}, 140 \mathrm{~m}$, and $200 \mathrm{~m}$. The vertexes of the spherical modules are positioned onto a parabola. Each module is individually oriented such as its axis matches the normal to the parabola. The resulting focal length of the heliostat is close to the distance to the receiver aperture. The value for the reflectance of the mirrors is taken at $\rho=0.9$. The flux distribution delivered by the whole heliostat is the addition of the flux given by each of the nine modules.

$\operatorname{FLUX}_{H F}(x, y)=\sum_{M=1}^{M=9} \frac{P_{M}}{2 \pi \sigma_{H F}^{M^{2}}} e^{-\frac{\left(\left(x-x_{I}^{M}\right)^{2}+\left(y-y_{I}^{M}\right)^{2}\right)}{2 \sigma_{H F}^{M^{2}}}}$

where $P_{M}$ is the power delivered by one module of the heliostat, and $\sigma_{H F}^{M}$ is the effective HFCAL deviation associated to the module calculated with Eq. (3), $\left(x_{I}^{M}, y_{I}^{M}\right)$ the coordinates of the impact of the ray reflected by the center of the module on the receiver. This methodology for the optical simulation of the heliostat through the calculation of the flux given by its nine modules validates the fact that we fixe the tracking error $\sigma_{t}$ equal to $0 \mathrm{mrad}$ in both azimuth and elevation for the calculation of $\sigma_{H F}^{M}$ but we use it for the calculation of the coordinates of the impact point $\left(x_{\mathrm{AP}}, y_{\mathrm{AP}}\right)$.

In both SOLTRACE and HFCAL methods, each heliostat is defined by the parameters related to its nine modules: shape, size, coordinates and orientation. We compare those two models using the example of heliostat A15, whose parameters are indicated in Table 1. The position of this heliostat in the solar field is shown in Fig. 3.

The study compares, as an example, simulation results for the whole day of June 21.

The results of both SOLTRACE and HFCAL simulations are compared as follows: the flux density distribution delivered by the heliostat A15 is computed by SOLTRACE and by HFCAL at 8 am, $10 \mathrm{am}, 12 \mathrm{pm}, 2 \mathrm{pm}, 4 \mathrm{pm}, 6 \mathrm{pm}$ (solar time). during the day June 21. At each time the power intercepted by a flat circular aperture of given diameter is extracted from the flux distribution. The number of rays for the SOLTRACE's ray tracing algorithm is fixed to 500,000 . The variation of the intercepted power versus the size of the aperture is plotted on Fig. 4 for both SOLTRACE and HFCAL simulated results.

As depicted in Fig. 4 the power intercepted by a circular aperture of growing area is quite similar for both 

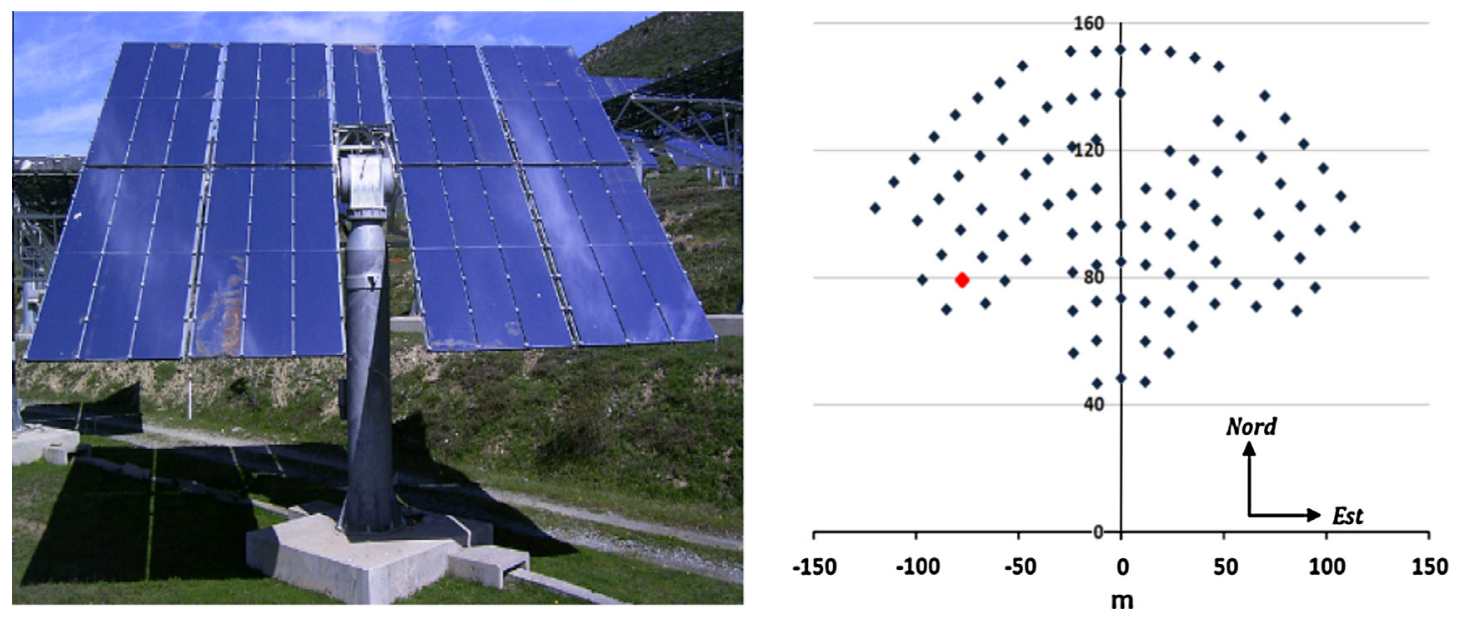

Fig. 3. Picture of one heliostat of the THEMIS solar field (left) and position of heliostat A15 in the field (right).

SOLTRACE and HFCAL models. The study of Fig. 4 shows us a less than $5 \%$ mean deviation for the power density distribution in any cases. Fig. 5 presents calculated flux density distribution maps for heliostat A15 at 8 am and $12 \mathrm{pm}$ (as seen from the front of the aperture). The main difference between the distributions given by the two models is due to the fact that HFCAL model consider a circular distribution while SOLTRACE, which uses a ray tracing method, permit to consider asymmetric deformations. The two models estimate similar peaks flux values: 16.7 and $15.3 \mathrm{~kW} / \mathrm{m}^{2}$ at $8 \mathrm{am}$ for respectively SOLTRACE and HFCAL model, and 15.0 and $13.6 \mathrm{~kW} / \mathrm{m}^{2}$, at $12 \mathrm{pm}$. The proximity of those results leads us to consider that HFCAL is accurate sufficiently for the flux density distribution optimization process and reliable to simulate the flux density distribution of one heliostat. This proximity can be explained by the fact that the same methodology is used in both model for the simulation of the sun shape (distribution of the solar irradiance) and tracking and optical errors (through a circular normal distribution for the normal to the reflecting surface). Thus, the main difference between those two models is due to the off-axes reflections deformations treatment.

HFCAL and SOLTRACE are then used to simulate the flux density distribution delivered by the entire solar field (107 heliostats) on June 21 at $9 \mathrm{am}, 12 \mathrm{pm}$, and $3 \mathrm{pm}$ (solar time). for a single centered aiming point strategy. The respective flux distributions at $12 \mathrm{pm}$ are plotted on Fig. 6, featuring peak flux density of 2603 suns and 2705 suns for respectively HFCAL and SOLTRACE. This flux density deviation of $3.4 \%$ between the two models shows us that on the whole field of heliostats, HFCAL slightly overestimates the flux density. We consider the results close enough for using HFCAL instead of SOLTRACE. HFCAL method is by far faster than ray tracing methods. HFCAL computes the flux density distribution on the receiver plane for the entire solar field in around $10 \mathrm{~s}$. It is then suitable for an optimization process which requires at each iteration step the computation of the flux map delivered by the complete solar field.

\section{Optimization process}

We address a general problem in which the receiver is represented by the aperture (receiver without cavity). The detailed design of the receiver shape and area is ignored. This approach is relevant since no measurement of flux density or temperature is done on the receiver surface. We propose an open loop strategy using HFCAL code to predict the flux distribution on the aperture in a fast, accurate and reliable computation step. The objective is to flatten as much as possible the distribution of flux density on the aperture plane and to minimize in the same time the spillage. The optimized aiming point strategy (OAPS) is based on the selection of a set of aiming points on the aperture plane and on the implementation of the generic algorithmic TABU meta-heuristic method in the solver to attribute at every time one aiming point at each heliostat. An adaptation of TABU is then proposed to improve this optimization process.

\subsection{The optimization problem}

Seeking for a combination of heliostats aiming at predefined points in the aperture plane is a discrete process with combinatorial characteristic. The size $|S|$ of the solutions space is expressed by:

$|S|=n_{\mathrm{AP}}^{n_{\mathrm{Hel}}}$

where $n_{H e l}$ is the number of heliostats and $n_{\mathrm{AP}}$ is the number of aiming points.

A part of the optimization problem consists of defining the appropriate number and distribution of aiming points on the aperture. The number and the distribution of aiming points are related to the size of the aperture. They also impact the flux map resolution and the computation time. Adding one aiming point in the mesh strongly increases the number of solutions. But it might yield relevant solutions as well.

Regarding the theoretical complexity, OAPS appears to be NP-HARD. We observe many similarities with 
Table 1

Parameters of heliostat A15.

\begin{tabular}{|c|c|c|c|c|c|}
\hline \multicolumn{3}{|c|}{ Position of the heliostat in the field (origin: bottom of tower) } & \multirow{2}{*}{$\begin{array}{l}\text { Focal length of } \\
\text { modules }\end{array}$} & \multirow{2}{*}{$\begin{array}{l}\text { Focal length of } \\
\text { heliostat }\end{array}$} & \multirow{2}{*}{$\begin{array}{l}\text { Distance to receiver } \\
\text { aperture }\end{array}$} \\
\hline East & North & Elevation & & & \\
\hline$-76.9 \mathrm{~m}$ & $78.9 \mathrm{~m}$ & $17.4 \mathrm{~m}$ & $140 \mathrm{~m}$ & $124.2 \mathrm{~m}$ & $122.9 \mathrm{~m}$ \\
\hline
\end{tabular}

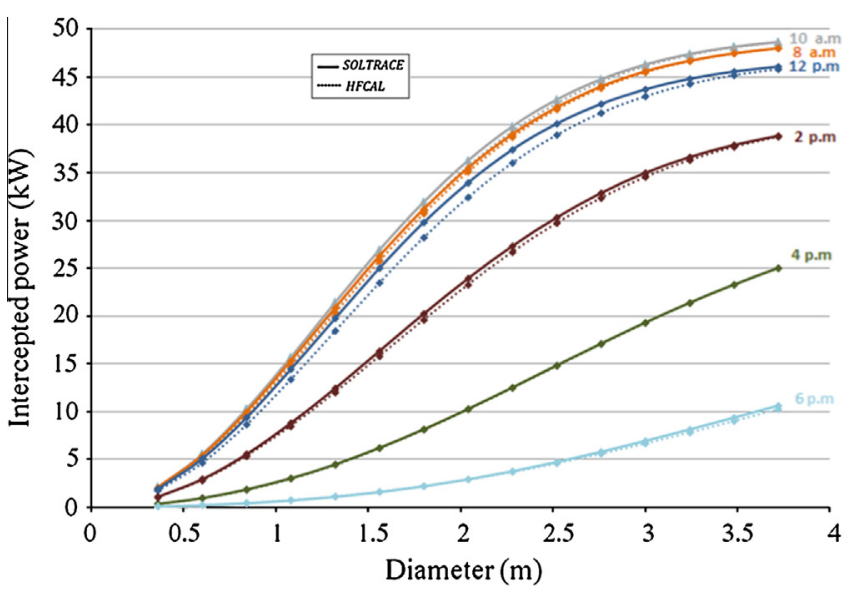

Fig. 4. Comparison between SOLTRACE and HFCAL models, heliostat A15 of THEMIS field, on June 21 at $8 \mathrm{am}, 10 \mathrm{am}, 12 \mathrm{pm}, 2 \mathrm{pm}, 4 \mathrm{pm}$, $6 \mathrm{pm}$ (solar time). Power intercepted by a flat circular aperture on the receiver with increasing diameter.

well-known academic problems like KNAPSACK (Garey and Johnson, 1979). We assume that finding and guaranteeing the global optimal solutions among large data can be intractable.

In the case of a homogenization of the flux density on the whole aperture plane, two criteria appear to be sufficient for the optimization:

- The spread of flux density on the aperture, which must be minimized.

- The part of the power reflected by the solar field which is intercepted by the aperture, which must be maximized.

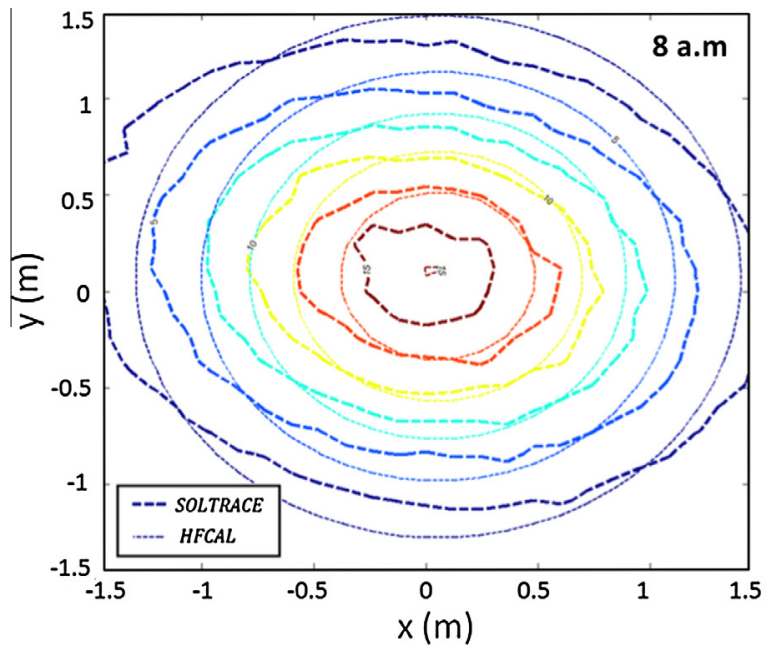

These criteria are represented on the sketch of the aperture plane in Fig. 7.

In the case of the objective of a non-homogenized flux density distribution on the aperture plane, the standard deviation regarding a desired local value of flux concentration must be used instead of the spread of flux density. Moreover, if a thermodynamic model of the receiver exists, it might allow defining laws of conversion between local flux densities and local efficiencies of the receiver. Thus, the global efficiency of the receiver can be calculated and be used as the main optimization criteria. This case is not treated in this paper. However, results of optimization algorithms using either spread of flux density, or standard deviation of flux density, or receiver efficiency are similar for the test-case of an homogenization of the flux density on the whole aperture plane which is treated in this paper.

A couple of parameters are used to quantify the quality of a solution. The spread quantifies the uniformity of the flux distribution on the aperture:

Spread $=F_{\max }-F_{\min }$

where $F_{\max }$ and $F_{\min }$ are respectively the maximum and the minimum flux concentration on elements of the mesh grid of the discrete receiver. The spread is used in the algorithm as the quality function (also called objective function). Its value must be minimized at any time.

The incremental added spillage $(S L)$ :

$S L=1-\frac{P_{r e c}}{P_{r e f}}$

where $P_{r e c}$, and $P_{r e f}$ respectively, is the power intercepted by the aperture with, and respectively without, aiming

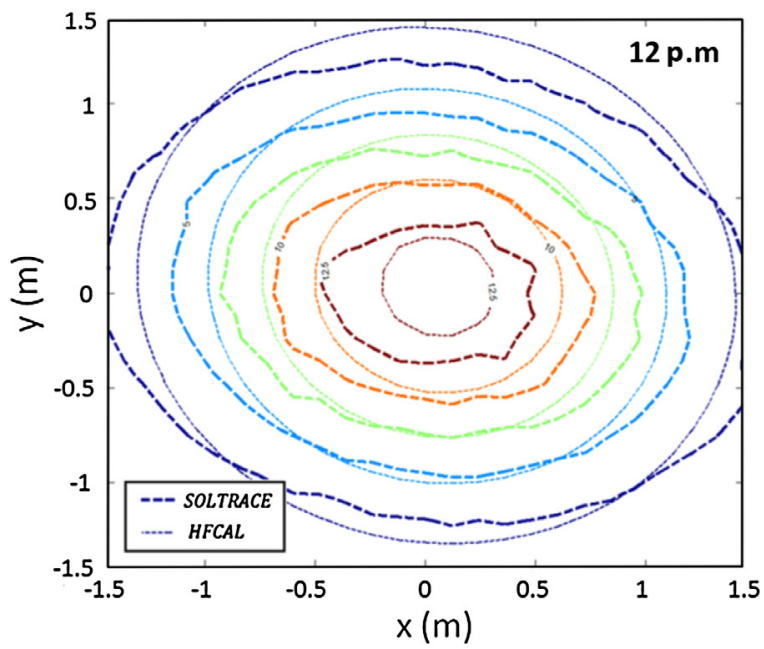

Fig. 5. Flux density distribution for heliostat A15 of the field on June 21 at 8 am and $12 \mathrm{pm}$, simulated by HFCAL and by SOLTRACE (500,000 rays). 

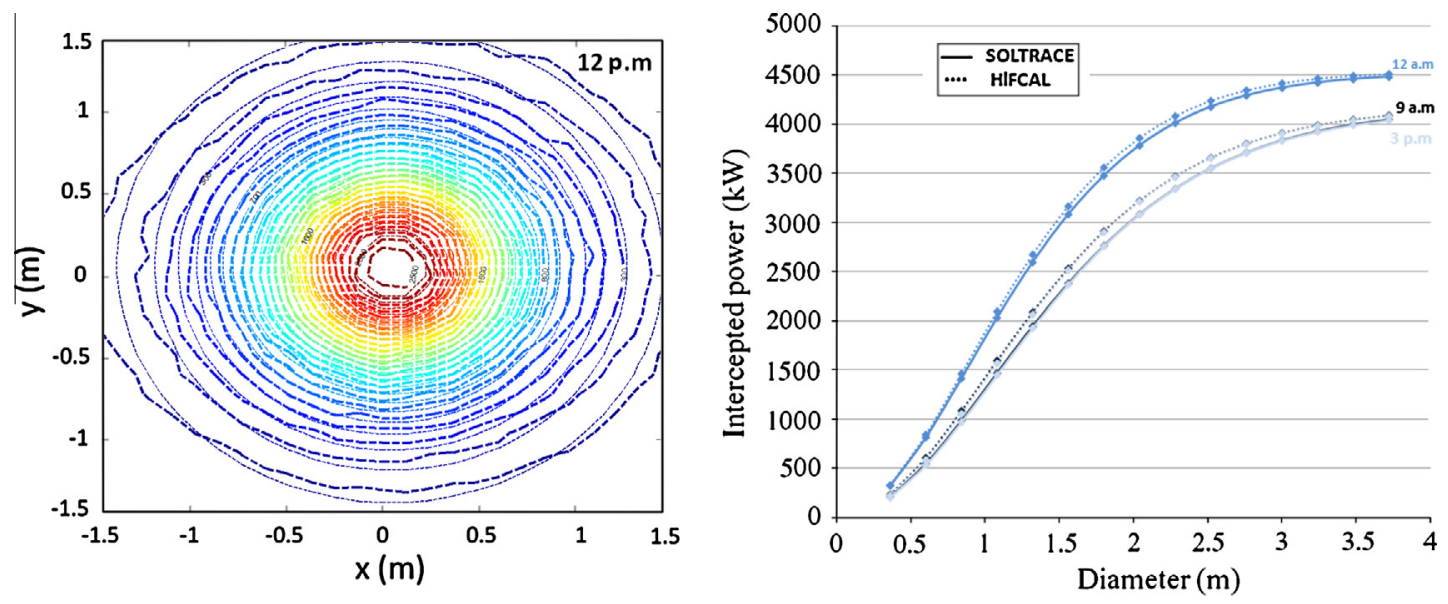

Fig. 6. (left) Comparison of the flux distribution simulated by the SOLTRACE (500,000 rays) and the HFCAL models for the entire THEMIS solar field, on June 21 at solar time $12 \mathrm{pm}$ (right). Power intercepted by a flat circular aperture on the receiver with increasing diameter.

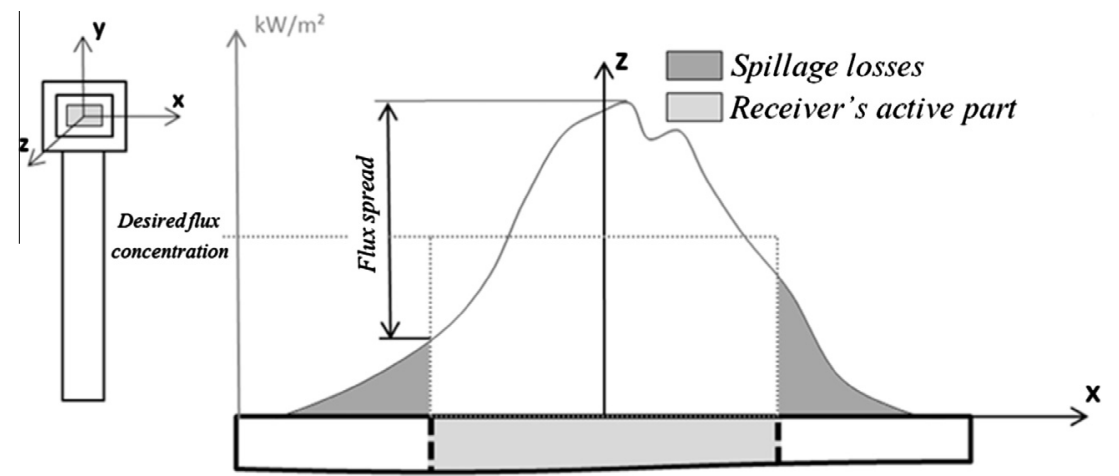

Fig. 7. Sight view of the aperture plane. Represented here are the optimization criteria: the flux spread on the aperture and the spillage losses.

point strategy. $P_{r e f}$ is the reference intercepted power. The value of $S L$ must remain lower than a threshold in the algorithm.

The spread decreases when the aiming points are distributed all over the aperture, but the spillage increases. Many computer-assisted methods are conceivable to find an acceptable trade-off between these contradictory objectives. We have selected the TABU meta-heuristic method and we have implemented it in the solver code.

\subsection{TABU meta-heuristic}

For OAPS, one solution is a vector of $n_{\mathrm{Hel}}$ pairs. Each pair is made of one heliostat associated with one aiming point. The TABU meta-heuristic was developed by Glover and McMillan (1986). It is a local search method. A local search method is a stochastic method which explores the solution configuration space by modifying the last solution. A neighborhood is defined as the set of solutions that are

\begin{tabular}{|c|c|c|c|c|c|c|}
\hline & H1 & $\mathrm{H} 2$ & H3 & $\mathrm{H} 4$ & H5 & H6 \\
\hline AP 1 & $S_{1,1}$ & $\mathrm{~S}_{1,2}$ & & $S_{1,4}$ & $S_{1,5}$ & $\mathrm{~S}_{1,6}$ \\
\hline AP 2 & $S_{2,1}$ & $S_{2,2}$ & $S_{2,3}$ & $S_{2,4}$ & $S_{2,5}$ & \\
\hline AP 3 & $S_{3,1}$ & 3, & $S_{3,3}$ & & $S_{3,5}$ & $S_{3,6}$ \\
\hline AP 4 & $\gamma_{4,1}$ & $S_{4,2}$ & & $\mathrm{~S}_{4,4}$ & & $S_{4,6}$ \\
\hline AP 5 & $S_{5,1}$ & $S_{5,2}$ & $S_{5,3}$ & $S_{5,4}$ & $S_{5,5}$ & $S_{5,6}$ \\
\hline
\end{tabular}

Fig. 8. Heliostat/aiming point matrix. Solution at iteration $i+1$ is reached from the solution at iteration $i$ by changing one heliostat/aiming point couple. 
reached when all the different accepted modifications are made.

At each iteration step, one heliostat is randomly selected. By changing its aiming point, a new solution is computed. This new solution differs from the previous one only by one pair heliostat/aiming point. HFCAL computes the distribution of flux density on the aperture plane with the new configuration. If the flux distribution is improved (through the objective function) the configuration is saved and the solver jumps to the next iteration step. As an example, Fig. 8 illustrates the case of a solar field of only six heliostats and five aiming points distributed on the aperture plane.

In TABU the solver avoids confinement in a local optimum. When no improvement of the objective function is obtained by any change in one step, the solver authorizes several changes with no improvement. In addition, one heliostat cannot return back to its previous aiming points during several steps. The changes made at every step are recorded. The solver scans all the directions in order to escape from a local optimum.

In the example showed on Fig. 8, if the heliostat H3 moves from aiming point AP 1 to aiming point AP 4, it will not move back from AP 4 to AP 1 during a fixed number of iterations.

When an optimum is identified, the solver restarts the process from a new randomly selected initial configuration. A new region of the solutions space is then explored.

TABU algorithm looks for the local optimum in this region. A fairly good trade-off is achieved between the
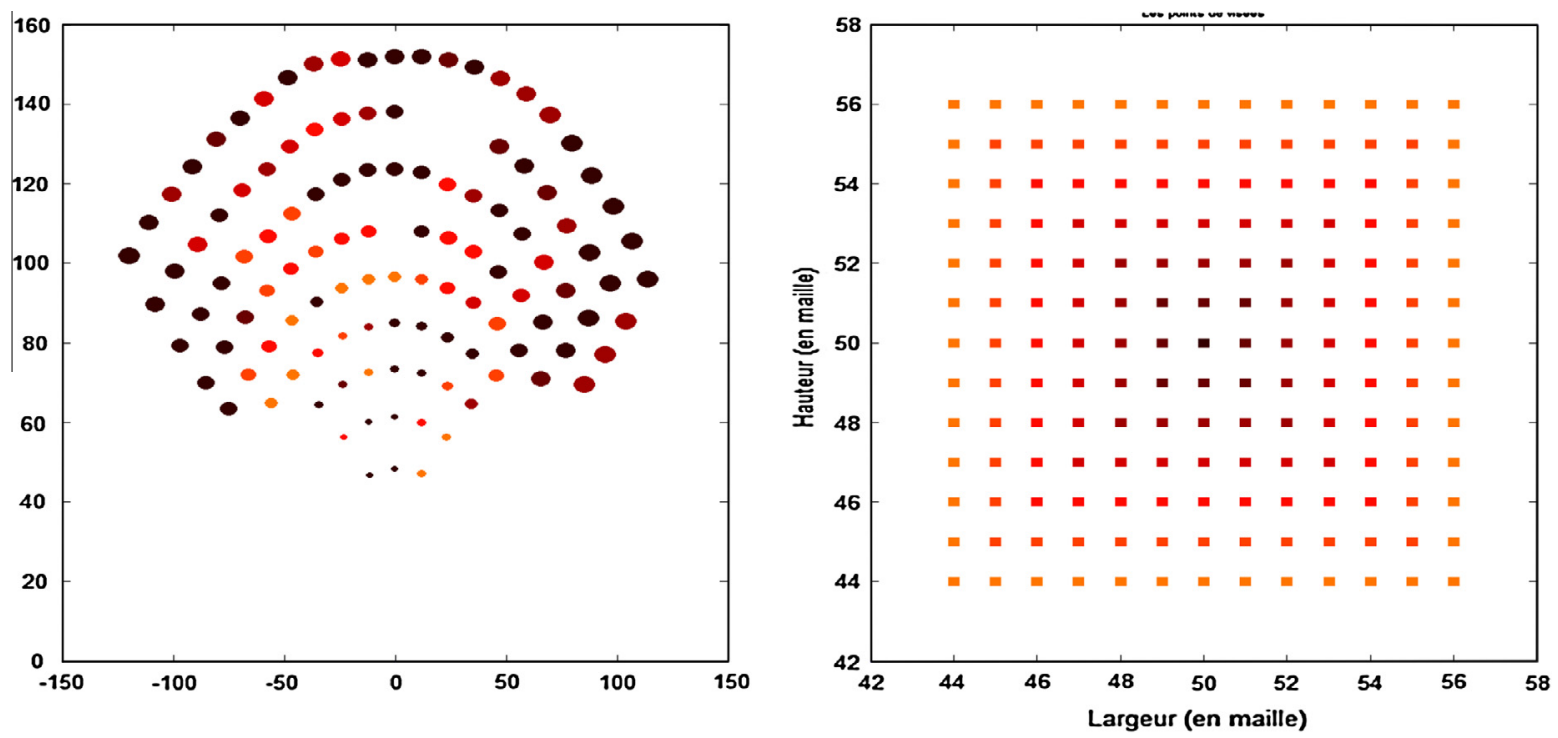

Fig. 9. Example of solution provided by the improved TABU algorithm. The colors of the heliostats' markers (left) are linked to the aiming points (right).

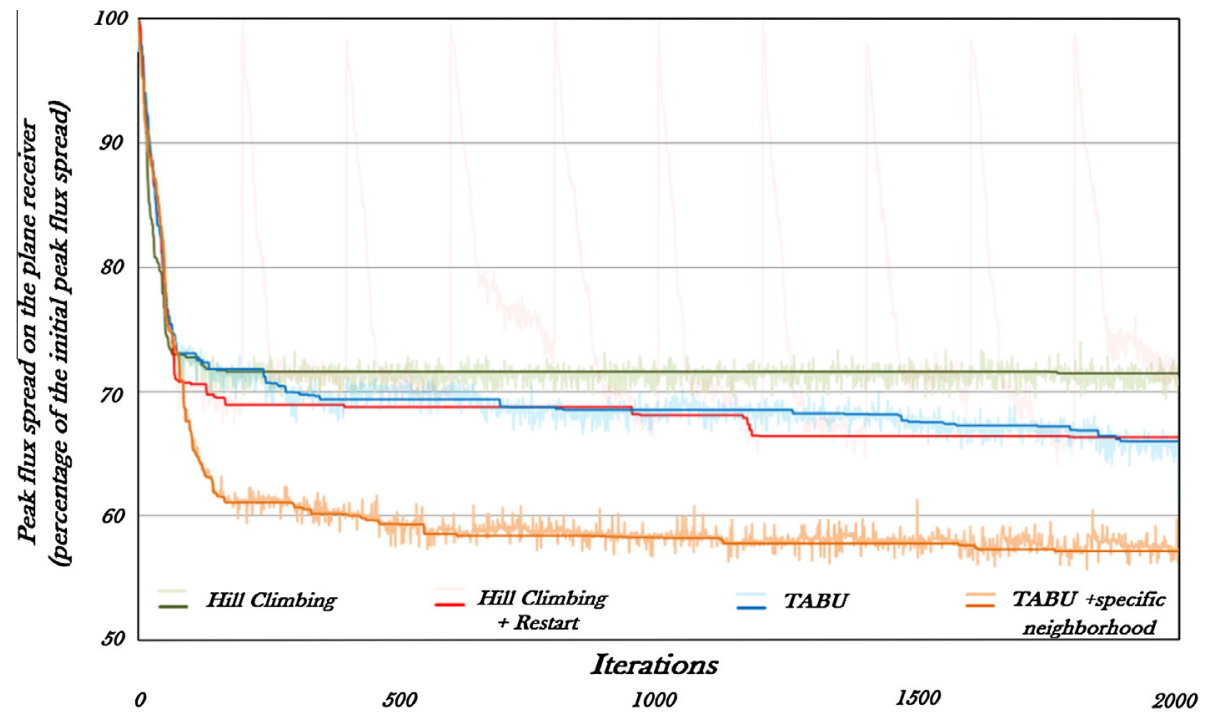

Fig. 10. Evolution of the spread of flux density on the $2 \mathrm{~m} \times 2 \mathrm{~m}$ aperture area, computed with four different algorithms. Test case: June 21 at 11 am (solar time). The threshold for spillage losses is $10 \%$ above the reference value. In fine lines: step by step results. In bold lines: average results. 
(a) 25 aim points

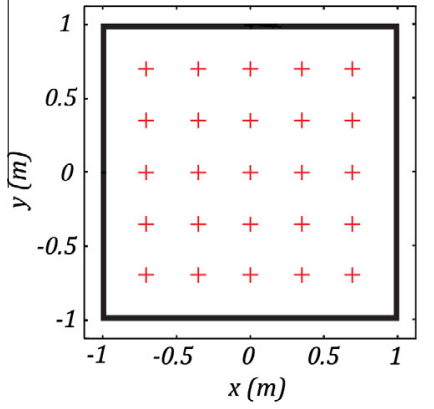

(b) 49 aim points

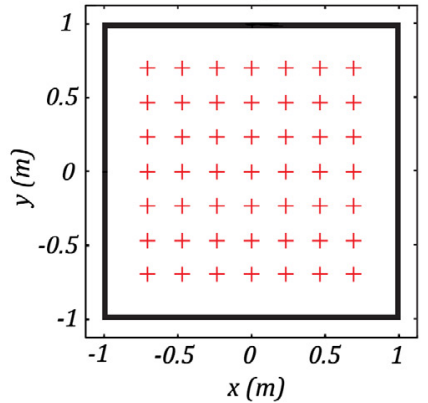

(c) 169 aim points

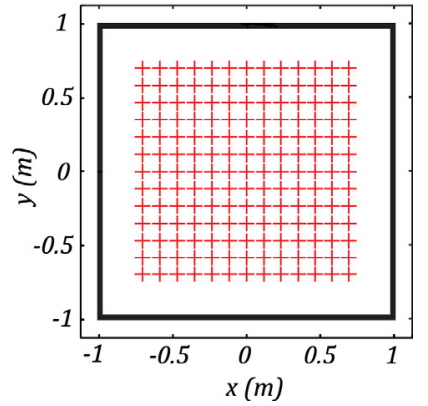

Fig. 11. Uniformly distributed aiming points on the $2 \mathrm{~m} \times 2 \mathrm{~m}$ square aperture.
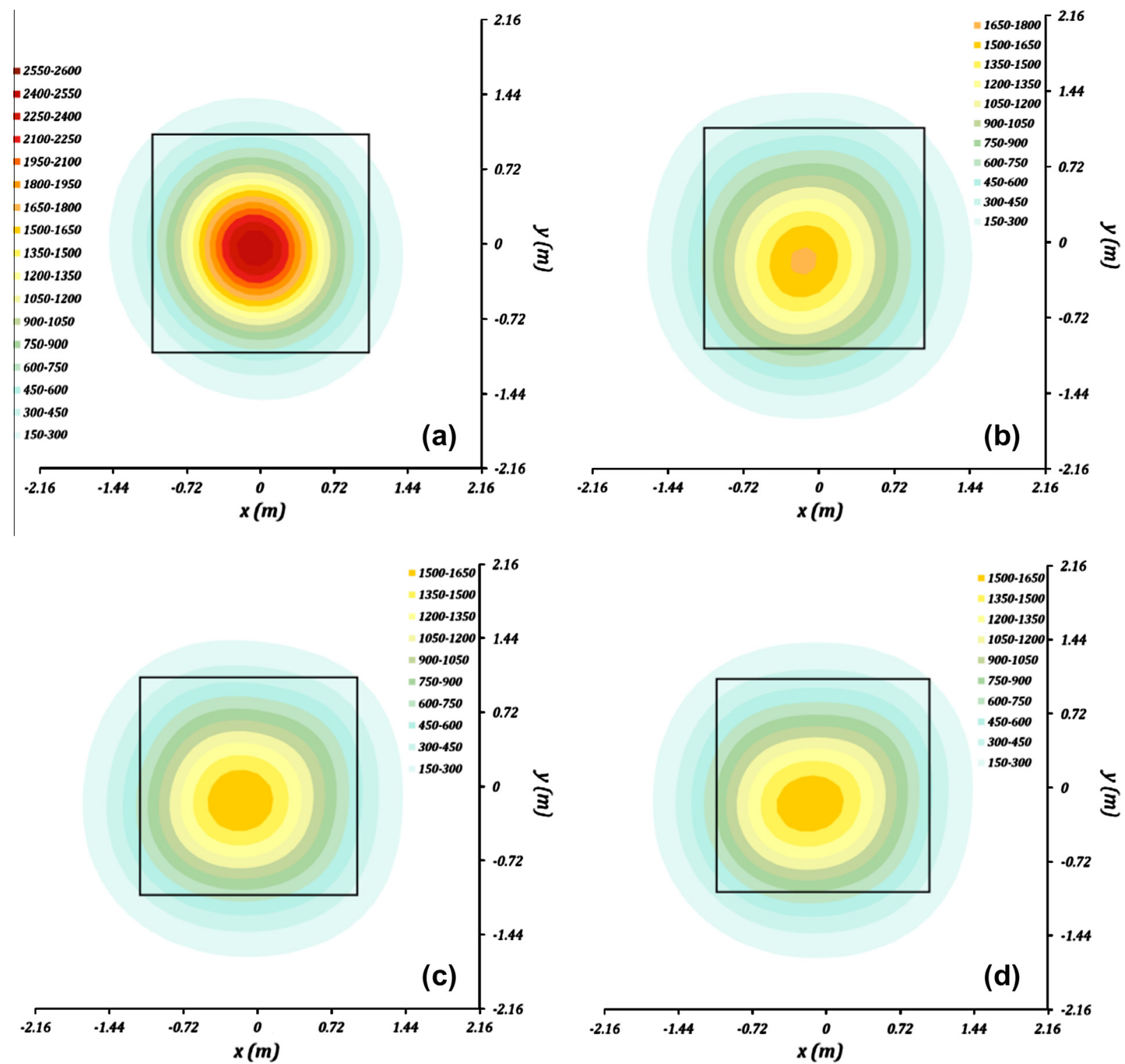

Fig. 12. Simulated distribution of concentration ratio on June 21 at 11 am (solar time). Without aiming point strategy (a) and with optimized aiming point strategies using various aiming point grids, e.g. 25 points (b), 49 points (c) and 169 points (d). The square represents the $2 \mathrm{~m} \times 2 \mathrm{~m}$ aperture. The maximum tolerated added spillage losses for the aiming points strategies is $10 \%$ (percent of the initial power on the aperture).

two main goals for an optimization algorithm: diversification (sampling) and intensification (quality).
Each iteration step consists of changing the aiming point of one heliostat. Therefore the velocity of this meta-heuristic 
is independent from the number and the density of aiming points and from the number of heliostats.

\subsection{Improved $T A B U$ algorithm}

In a solar field, the heliostats do not contribute to the concentration ratio on the target with the same optical efficiency at every time. The concentrated flux density delivered by one heliostat depends on its position in the field related to the location of the target - efficiency decreases with increasing distance to the target- and to the position of the sun in the sky - efficiency decreases with increasing incidence angle (cosine effect). Heliostats located at the edge of the field offer lower optical efficiency than those located in the center of the field. An improvement of the solver is proposed. It consists of forcing low rated concentration heliostats to aim at aiming points located in the central area of the aperture. Spillage is therefore significantly decreased, while spread of the flux distribution is not increased dramatically.

This improvement is implemented in TABU through the definition of a specific neighborhood for the optimization problem. In practice, we attempt to restrict the solution space by forbidding solutions with a poor objective function, or to decrease the probability to explore them. The new neighborhood sets restrictions on available aiming points for low rated concentration heliostats: aiming points located at the edge of the aperture area are forbidden. Inversely, high rated concentration heliostats are allowed to aim at all the aiming points defined in the mesh.
An illustration of this method is given in Fig. 9. The solution provided by the solver is presented. On the left, every heliostat is represented by one marker. The size of the marker is proportional to the size of the image formed by the heliostat onto the aperture. The color of the marker is linked to its corresponding aiming point as defined on the right by the improved TABU algorithm. Low rated concentration heliostats aim at dark centered aiming points while the others aim at any points distributed over the entire mesh $(13 \times 13=169$ aiming points in this case $)$.

\section{Results and discussion}

\subsection{Compared algorithms}

The improved TABU algorithm is compared to three conventional local search meta-heuristic methods. The solutions are established on June 21 at solar time 11 am 25 aim points are defined on the square aperture of $2 \mathrm{~m} \times 2 \mathrm{~m}$ as defined in Fig. 12c. The excess for spillage losses is limited at $10 \%$ above the reference spillage (where no aiming point strategy is applied). The spread of the flux density distribution is plotted versus the number of iterations in Fig. 10. Four different algorithms are compared:

The first one is a simple local search Hill Climbing (Russell and Norvig, 2003). As depicted in Fig. 10, it quickly reaches a local optimum which in this case reduces the spread by $27 \%$ from the initial value. Since the local optimum is reached, the solution does not evolve anymore.

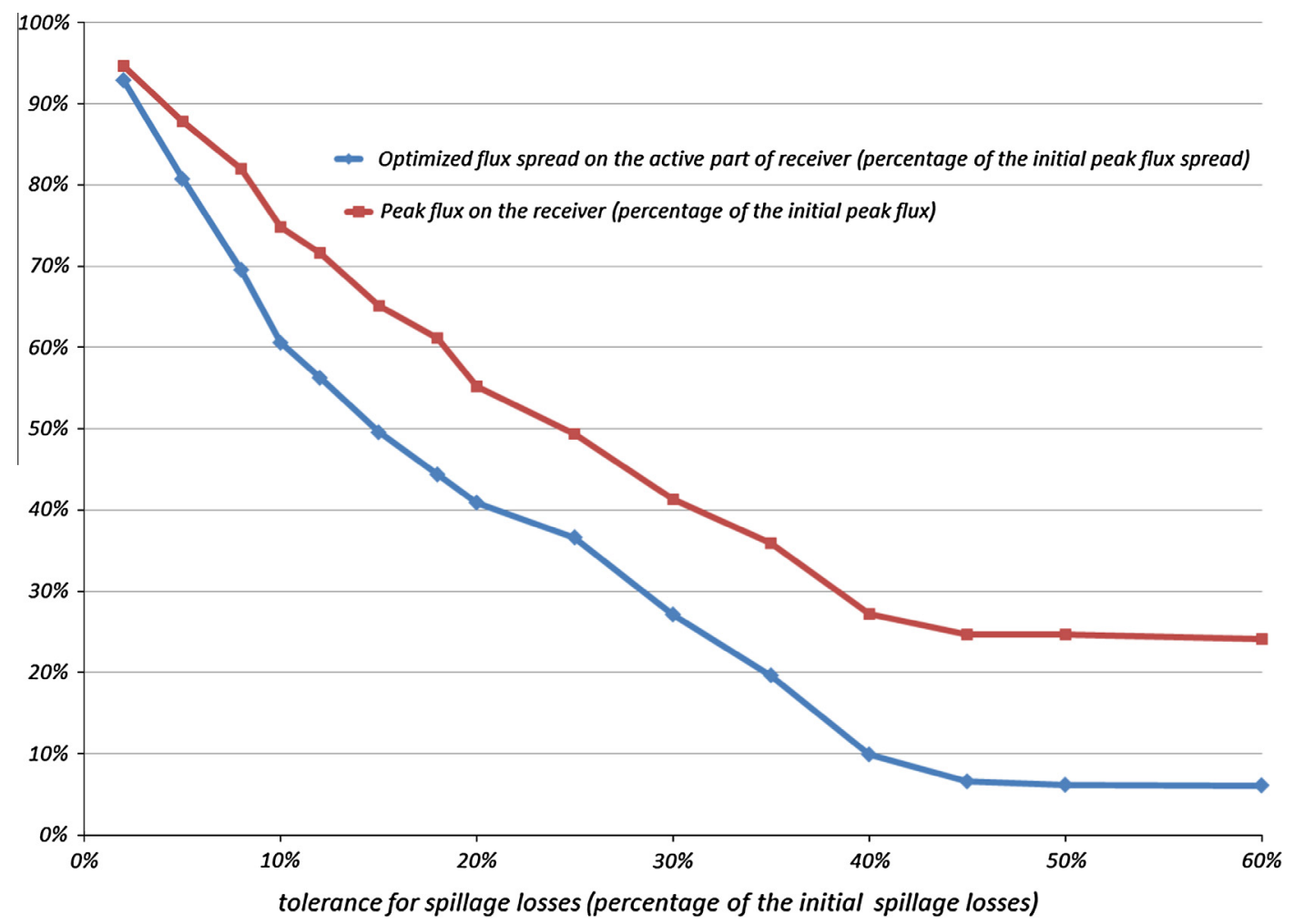

Fig. 13. Evolution of the spread of flux density and peak flux density with optimum aiming point strategy versus the accepted added spillage. The aperture is $2 \mathrm{~m} \times 2 \mathrm{~m}, 25$ aiming points; the day is June 21 at $11 \mathrm{am}$. 
The next algorithm is the Hill Climbing with an added "restart" procedure. When the solver considers that the quality function is not enough improved, it restarts the optimization process from the beginning. This method appears to be efficient as it uses a simple optimization to diversify the explored areas of the space of solutions. The spread of flux density is reduced by $32 \%$.

The third algorithm uses the TABU meta-heuristic described in Section 4.2. The solver tries to escape from any local optimum. The quality of the solution is similar to the previous one, as expressed through the spread of flux density which is reduced again by $32 \%$.

The last algorithm uses the improved TABU meta-heuristic described in Section 4.3. This method reduces the space of solutions by eliminating attempts which yield poor flux density distributions (elevated spread and/or big spillage). It appears to be the most efficient method as it quickly reduces the spread by about $39 \%$ and further yields an additional reduction down to $43 \%$.

As all those algorithms are based on local searches defined with the same movement (a heliostat/aiming point couple), the iteration time are thus similar with 100 iterations in less than $1 \mathrm{~s}$. And as it can be seen on Fig. 10, the evolution of the solution's quality slow down around the 2000th iteration (20 s) which mean that the most important part of the improvement is done and really acceptable solutions are reached.

\subsection{Influence of the aiming point grid density}

As it is indicated in Fig. 11, three different aiming point grids are defined onto the $2 \mathrm{~m} \times 2 \mathrm{~m}$ square aperture: 25 points $(5 \times 5), 49$ points $(7 \times 7)$ and 169 points $(13 \times 13)$. In all cases the points are uniformly distributed. The entire heliostat field is used and 10\% additional spillage loss is admitted above the reference value. The optimized aiming point strategy is obtained using the improved TABU algorithm. The case of June 21 at solar time 11 am is taken as example. The results are compared to the reference case in which no aiming point strategy is applied.

The computation time required by the improved TABU algorithm slightly increases with increasing number of aiming points. The distributions of concentration ratios simulated by HFCAL in the tested configurations are plotted in Fig. 12.

Without aiming point strategy (a), the peak concentration ratio is estimated at 2525 suns. The optimization process using improved TABU algorithm for the aiming point strategy onto 25 (b), 49 (c) and 169 (d) aiming points reduces this peak concentration down to 1677 suns (b), 1642 suns (c) and 1624 suns (d) respectively. The gain on the peak concentration resulting from the aiming point strategy is clearly established, even when a low number of aiming points are defined. The distribution of concentration is a little bit more flattened when the number of aiming points increases from 25 to 49 , and it remains almost

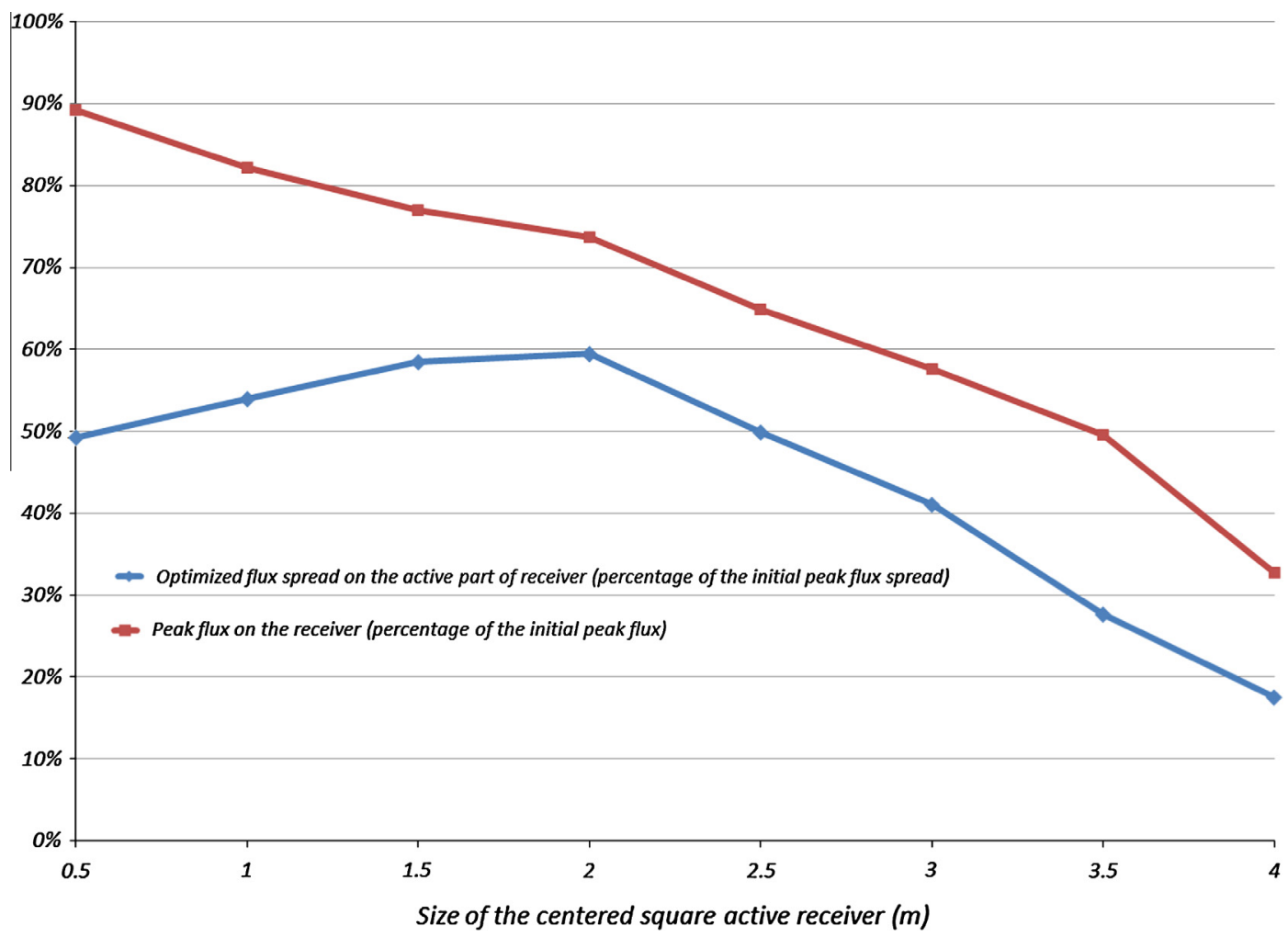

Fig. 14. Evolution of the spread of flux density and peak flux density with optimum aiming point strategy versus the size of the aperture. The day is June 21 at $11 \mathrm{am}$. The size of the square aperture is expressed by the length of one side $(0.5-4 \mathrm{~m})$. The maximum tolerated added spillage losses for the aiming points strategies is $10 \%$ (percent of the initial power on the aperture). 
unchanged when this number increases more. The utilization of a very dense aiming point grid is therefore useless. In our case, a grid of 25 aiming points appears to be enough. The main limiting factor for the density of the grid is the tracking error of heliostats. THEMIS heliostats have a $0.26 \mathrm{mrad}$ tracking error, moreover, the mean distance between heliostats and receiver is $126 \mathrm{~m}$. Thus the mean deviation of heliostats focusing point on the receiver plane is $3.3 \mathrm{~cm}$, which represented the upper threshold for grid density.

\subsection{Spillage}

How much does the accepted value of additional spillage impact the optimized solution? This is analyzed through the observation of the peak flux density and of the spread of flux density onto the aperture when the threshold for additional spillage increases, from $2 \%$ to $60 \%$ of the reference value. The optimization process uses 25 aiming points uniformly distributed on the $2 \mathrm{~m} \times 2 \mathrm{~m}$ square aperture (Fig. 12a). The variations of the peak flux density and of the spread of flux density related to their reference values are plotted in Fig. 13, versus the accepted additional spillage. When the accepted additional spillage increases up to $40 \%$, the peak flux density and the spread of flux density decrease linearly. A greater spillage does not improve the solution any more. The best solution for this grid regarding the distribution of flux density corresponds to an approximately $40 \%$ spillage losses. This optimum point can be different from one aiming point grid to another and from one study point to another; but the major report is that the tolerance for spillage losses is one of the most influent parameter for the optimization process as it linearly influences the solution quality with a strong slope, and it's one of the most intuitive to define for the user. The user has to carefully fix this parameter as it has a great impact on the solution quality, with respect to the spread of flux density, the peak flux tolerated by the receiver, and the desired amount of power on the receiver's aperture.

\subsection{Size of the aperture}

The spillage losses increase when the aiming point strategy defocuses more heliostats from the center to the edge of the aperture. The spillage losses also increase with decreasing size of the aperture. The influence of the size of the aperture on the quality of the optimized solution for the aiming point strategy is examined. The maximum accepted additional spillage is $10 \%$ above the reference spillage. The density of the aiming points on the aperture plane is kept equal to the density presented in Fig. 12b, e.g. the size of the mesh is $24 \mathrm{~cm}$ (distance between two neighbors). When the size of the aperture changes, the number of aiming points changes accordingly. For every aperture size, the solver optimizes the aiming point strategy using the improved TABU algorithm.
The variations of the peak flux density and of the spread of flux density related to their reference values are plotted in Fig. 14, versus the size of the aperture. For a very large aperture, the aiming point strategy yields low values of peak flux density and of spread of flux density (Fig. 15). When the size of the aperture decreases, the aiming point strategy becomes less efficient, the peak flux density increases. For an aperture smaller than $2 \mathrm{~m} \times 2 \mathrm{~m}$, the situation is different: the size of the concentrated beam becomes larger than the size of the aperture. The aiming strategy continues to increase the peak flux by focusing more heliostats in the center of the aperture, but the spread of the flux density decreases because the aperture intercepts only the central part of the flux density distribution which is almost flat. In the same time the spillage increases, the additional spillage cannot remain lower than $10 \%$. The aiming point strategy is no longer efficient. It is also meaningless. This clearly shows that the aperture should remain larger than the mean size of the concentrated beam at the aperture plane.

\section{Conclusion and perspectives}

The operator of a solar tower concentrating system often needs an aiming point strategy for improving the concentrated flux density distribution onto the target, which is generally the surface of the solar absorber or the aperture of the solar receiver. The goal is to flatten the flux density distribution while keeping the spillage within a reasonable range. In this work an open loop control system is proposed and is implemented in the THEMIS experimental solar tower installation (France). HFCAL code is the core of this system. It is a fast, reliable and accurate numerical simulation tool which computes flux density distributions onto the aperture. HFCAL model was validated against experimental flux distributions measured during a full moon night. It was also successfully compared to the raytracing model SOLTRACE. A complete comparison of HFCAL model with measurements on THEMIS site is the subject of a specific paper which is in development.

HFCAL model is combined with a solver which seeks for the best aiming point for each heliostat in an iterative process, until the objectives are achieved: minimum spread of flux density distribution and additional spillage lower than the given threshold. The TABU meta-heuristic algorithm was implemented in this solver. This heuristic quickly finds local optimum (it requires about 2000 iterations calculated in $20 \mathrm{~s}$ ), but it also prevents falling definitely into a local optimum. An improvement was proposed to this algorithm in order to restrict the space of solutions for the heliostats featuring a poor optical efficiency or a wide solar beam. These latter keep focusing at the central region of the aperture.

The operator defines the two competing objectives of the strategy. He sets the maximum values of the accepted additional spillage and of the spread of the flux density distribution as well as the number and the location of the aiming 

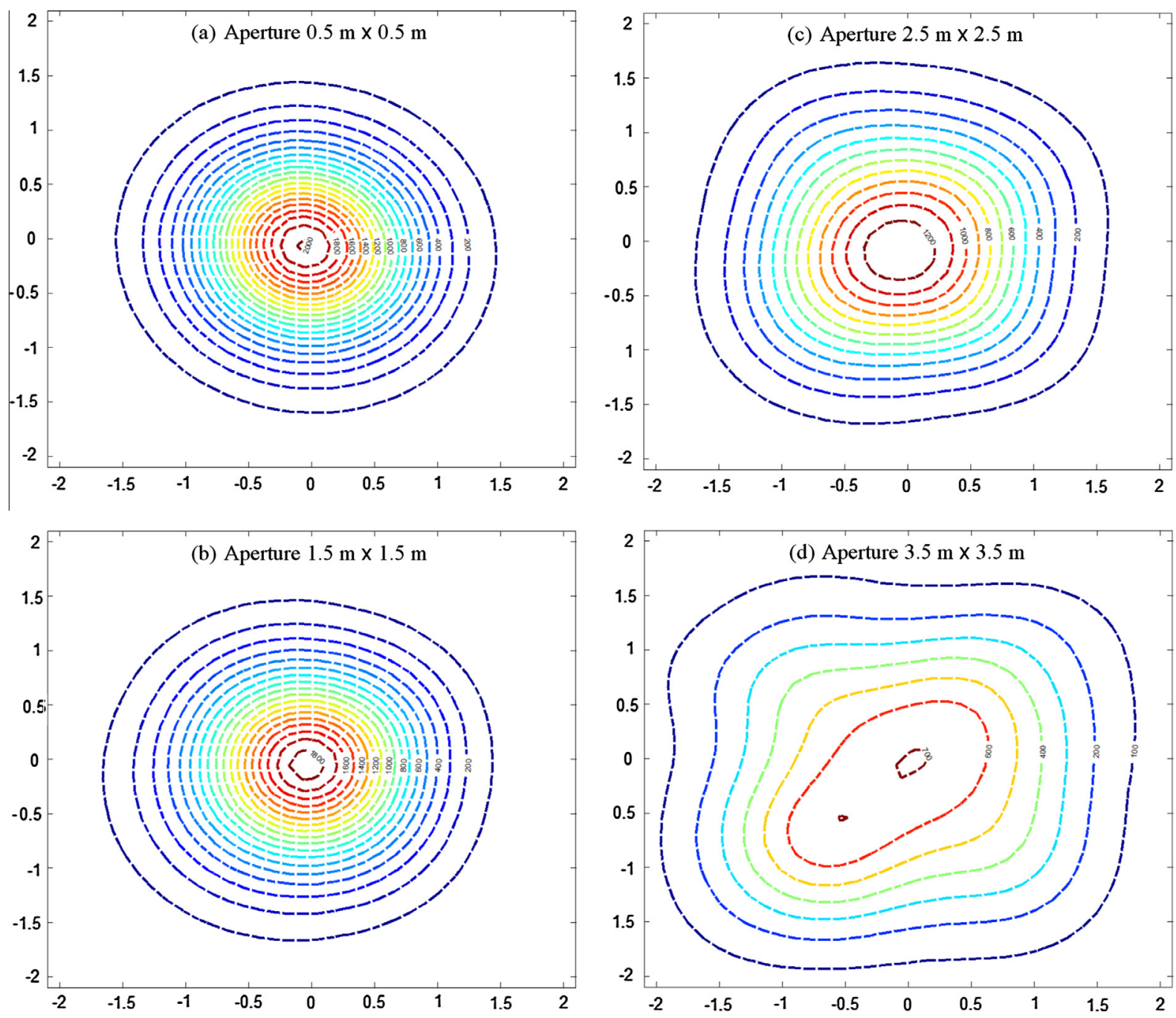

Fig. 15. Flux density distributions using the whole THEMIS heliostat field as computed by HFCAL for the optimized aiming point strategies with various sizes of aperture. Spillage loss threshold $=10 \%$.

points. This definition step is of prime importance for the execution of the optimization process but it can be achieve by the computer with respect to the mean size of heliostats spots, and the size of the aperture. Main advantages of TABU algorithm is that the convergence of the optimization process is assured, moreover, this algorithm can be easily calibrated as all its parameters have a physical correspondence (whereas for example bio inspired meta-heuristics require the definition of meta-parameters) and finally, iteration time is not dependant to the number of heliostat and aiming points.

The achievements of this work show that the distribution of flux density on the aperture plane is significantly flattened when the optimized aiming point strategy is used. With an added spillage of only $10 \%$, the spread of flux density is decreased by $43 \%$ referred to the spread observed without aiming point strategy. In comparison, other algorithms were tested, yielding to spread reduction of $32 \%$.

The number of aiming points is of small importance, providing that their distribution on the aperture plane is uniform and that they cover all the aperture area. The tests made with 25 aiming points distributed on a $2 \mathrm{~m} \times 2 \mathrm{~m}$ square aperture showed very good results. The peak flux density was reduced from 2525 suns down to 1677 suns. The improvement resulting from an increase of the number of aiming points up to 49 and 169 remained very small, yielding a reduction of peak flux density down to 1642 suns and 1624 suns respectively. Heliostats tracking errors seem to be the major limiting factor for aiming point grid density as the grid density does not increase significantly the calculation time and does not modify significantly the results.

Finally, it was confirmed that the size of the aperture (or the size of the active part of the receiver) clearly impacts the potential for the reduction of spread of flux density. The greater is the aperture, the more efficient is the aiming point strategy. On the other hand, the aperture should not be smaller than the minimum size of the concentrated beam. Otherwise the spillage dramatically increases and the aiming point strategy becomes meaningless.

The aiming point strategy proposed in this work is helpful for the operator. It might be later implemented into the 
Table 2

Parameters for HFCAL calculation.

\begin{tabular}{lll}
\hline$A_{m}$ & $53.85 \mathrm{~m}^{2}$ & Mirror area \\
$\rho$ & 0.9 & Reflectivity of the mirror \\
$\sigma_{\text {sun }}$ & $2.73 \mathrm{mrad}$ & Sun shape with standard deviation, \\
$\sigma_{b q}$ & {$[4]$} & Beam quality associated to mirror slope error \\
$\sigma_{\text {ast }}$ & {$[5]$} & Astigmatic effect (mrad) \\
$\sigma_{t}$ & $0.26 \mathrm{mrad}$ & Tracking error (mrad) \\
\hline
\end{tabular}

control software used for operating the solar field. Another future work might be the adaptation of the optimization process to non-flat receivers.

\section{Acknowledgments}

This work was partly funded by the French Ministry of Research, through the $\mathrm{PhD}$ research project of Adrien Salomé.

The authors also gratefully acknowledge Tim Wendelin and Allan Lewandowski from NREL for the development of SOLTRACE model and the tutorial for its implementation at PROMES laboratory. The authors also gratefully acknowledge J.M. Cormier for his contribution to the redaction of this article.

\section{References}

Garcia-Martin, F.J., Berenguel, M., Valverde, A., Camacho, E.F., 1999. Heuristic knowledge-based heliostat field control for the optimization of the temperature distribution in a volumetric receiver. Solar Energy 66 (5), 355-369.

Wendelin, T., 2003. SolTRACE: a new optical modeling tool for concentrating solar optics. In: Proceedings of the ISEC 2003: International Solar Energy Conference, 15-18 March 2003, Kohala Coast, Hawaii, New York, American Society of Mechanical Engineers, pp. 253-260.

Garcia, P., Ferriere, A., Bezian, J.J., 2008. Codes for solar flux calculation dedicated to central receiversystem applications: a comparative review. Solar Energy 82, 189-197.

Albert, R., 2009. Qualification optique des héliostats de Thémis. Internal note.

Schwarzbözl, P., Schmitz, M., Pitz-Paal, R., 2009. Visual HFCAL, a software tool for layout and optimization of heliostat fields. Solar Paces.

Collado, F.J., 2010. One-point fitting of the flux density produced by a heliostat. Solar Energy 84, 673-684.

Garey, M.R., Johnson, D.S., 1979. Computers and Intractability. W.H. Freeman, p. 247.

Glover, F., McMillan, C., 1986. The general employee scheduling problem: an integration of MS and AI. Computers and Operations Research.

Russell, Stuart J., Norvig, Peter, 2003. Artificial Intelligence: A Modern Approach. Upper Saddle River, pp. 111-114.

\section{Appendix A}

See Table 2. 